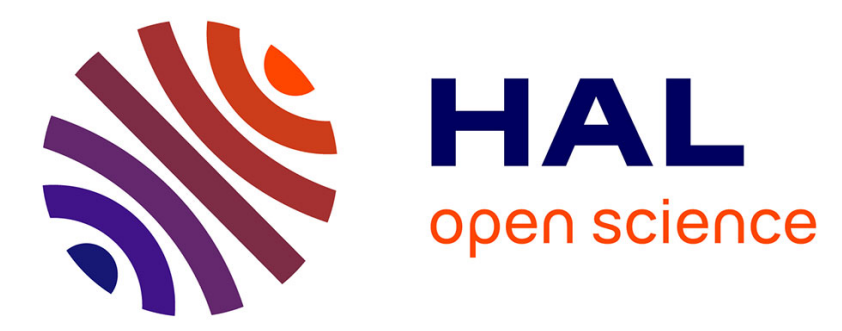

\title{
Rotation of a floating hydrophobic disk influence of line tension
}

\author{
Janine Emile, Hervé Tabuteau, Olivier Emile
}

\section{To cite this version:}

Janine Emile, Hervé Tabuteau, Olivier Emile. Rotation of a floating hydrophobic disk influence of line tension. Soft Matter, 2018, 14 (19), pp.3829-3833. 10.1039/c8sm00309b . hal-01806938

\section{HAL Id: hal-01806938 \\ https://hal-univ-rennes1.archives-ouvertes.fr/hal-01806938}

Submitted on 3 Jul 2018

HAL is a multi-disciplinary open access archive for the deposit and dissemination of scientific research documents, whether they are published or not. The documents may come from teaching and research institutions in France or abroad, or from public or private research centers.
L'archive ouverte pluridisciplinaire HAL, est destinée au dépôt et à la diffusion de documents scientifiques de niveau recherche, publiés ou non, émanant des établissements d'enseignement et de recherche français ou étrangers, des laboratoires publics ou privés. 


\title{
Rotation of a floating hydrophobic disk: influence of the line tension.
}

\author{
Janine Emile, ${ }^{1}$ Hervé Tabuteau, ${ }^{1}$ and Olivier Emile ${ }^{2, *}$ \\ ${ }^{1}$ IPR, UMR CNRS 6251, Université de Rennes I, 35042 Rennes Cedex, France. \\ ${ }^{2}$ Université de Rennes I, 35042 Rennes Cedex, France.
}

(Dated: April 16, 2018)

\begin{abstract}
The rotation of a sub-millimeter size disk over a water bath is reported. The origin of the rotation arises from the transfer of angular momentum from a plane wave diffracted by an asymmetrical picture printed on the disk. Because of its hydrophobic character, the viscous friction contribution to the rotational motion of the object floating on the air/liquid interface is weak. From the driving optical torque and the steady state rotation, we measure the contribution of the line tension, in the femto newton range.
\end{abstract}


The ability to rotate objects at the micrometer scale offers important applications[1, 2] ranging from microelectro-mechanical systems (MEMS) [3], microfluidic devices[4], biomanipulation such as torque induced spectroscopy[5], environmental remediation [6] or micro-rheology[7, 8]. Light induced torque has a special status among these applications, since it is generated from remote sources, without any contact or perturbation to the system, which may be detrimental, particularly in biological systems. The angular momentum can be transferred using specially dedicated three dimensional particles inspired from windmills[9], or via spin[10] or orbital angular momentum[11] transfer from light to matter. The advantage of the latter is that the transfer can be very efficient since, contrarily to the other mechanisms, each photon may carry several $\hbar$ momentum[12], $\hbar$ being the reduced Planck constant. In this last case however, since the typical size of the beam can be larger than the particle size, particles may rotate around the beam axis, having a circular trajectory, instead of spinning around their own axis, as expected. We have recently shown that orbital angular momentum can be transferred from a plane wave to an asymmetrical macroscopic object, because of diffraction[13]. The objects are then necessarily spinning around their own axis. One may then wonder whether such transfer can be performed at a microscopic scale. The aim of this communication is to study the rotation of a sub-millimeter disk over which an opaque diffracting asymmetrical disk is printed and illuminated by a plane wave, and to investigate its interfacial rotational properties.

The experimental set-up is depicted on figure 1 . The light originates from an infra red laser source (IDIL, $P=150 \mathrm{~mW}, \lambda=980 \mathrm{~nm}$ ) connected to a monomode optical fiber. The light is then focalized near the air/liquid interface with a $f=50$ mm-focal distance lens. The beaker is a $50 \mathrm{~mm}$-diameter cylinder. A thin glass plate acts as a cover and prevents from external vibrations, air flows and from excessive liquid evaporation. The height of the liquid inside the beaker is $4 \mathrm{~mm}$. We have checked that the water height has no influence on the experimental results. 


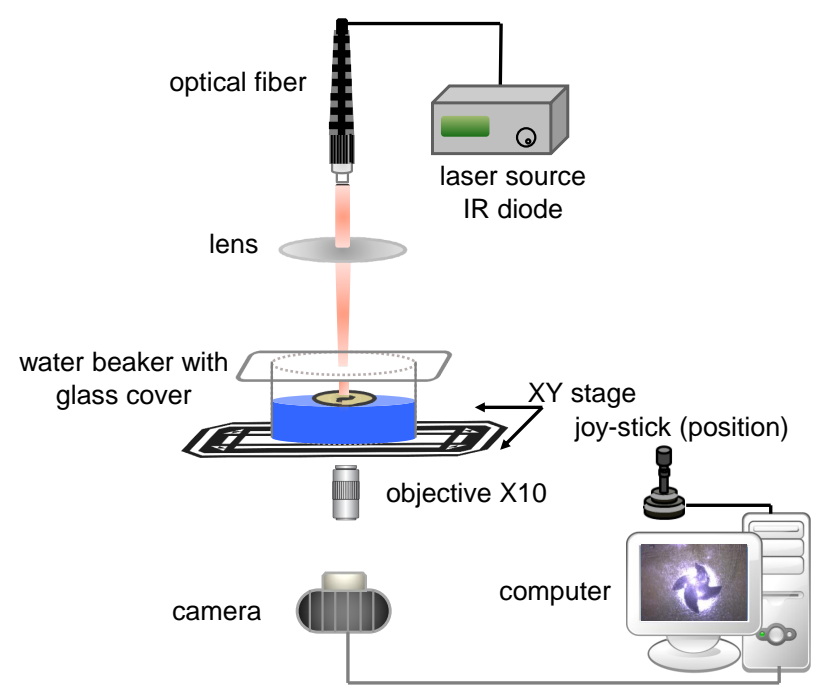

FIG. 1. Experimental set-up. Light source: diode laser at $\lambda=980 \mathrm{~nm}$ connected to an optical fiber. The images of the rotation are collected via a microscope and a camera connected to a computer.

The micrometer-size asymmetrical shapes are printed on a transparent hydrophobic polyester PET base with a soft photographic emulsion gel (JD Photo Data, thickness $187 \mu \mathrm{m}$, density $\left.\rho=1.37 \mathrm{~g} / \mathrm{cm}^{3}\right)$, then cut with a puncher which radius $R$ ranges from 0.5 up to $2.5 \mathrm{~mm}$. Note that the cutting process leads to notched disks. These diameters are larger than the asymmetrical printed pictures. The pictures that act as micro-motors are $500 \mu \mathrm{m}$ (typical size) paddle wheels with a number of wheels varying from $p=1$ to $p=6$ (see figure 2). However, since the rotation effects are more spectacular for high values of $p$, we don't discuss the $p=1$ to $p=3$ cases [13]. Whatever the considered liquid and the disk size, the surface tension is higher than gravity, and the disk is floating on the air/liquid interface. The object is imaged with an inverted microscope. Its position can be adjusted with a 2-D microstage that is used to track it. In order to optimize the transfer efficiency, the beam waist corresponds to the radius of the printed objects.

After cutting, the objects are rinsed with demineralized water and deposited on 


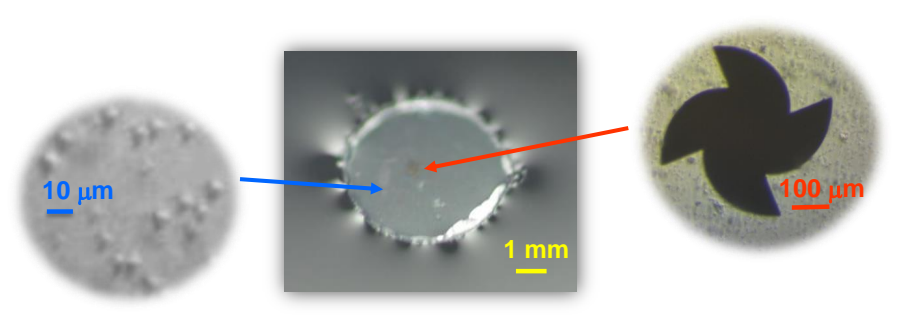

FIG. 2. Picture of the hydrophobic disk. Left: zoom showing the micro-air-bubbles. Right: zoom of the paddle wheel $(p=4)$.

the air/liquid interface. We then wait for 2 minutes till the object stops moving. The disk is then rotationally and translationally stable. Each object is used 6 times at most, before being wasted, because it degrades rapidly. The liquid is changed with the object. Each experiment is repeated 3 times at least with different objects in order to achieve a good experimental reproducibility. The experiment lasts for 3 minutes for large objects, and for $30 \mathrm{~s}$ for $R<1.0 \mathrm{~mm}$. For longer times, there are heating and temperature heterogeneities within the air/liquid interface. Since the paddle wheel is never exactly at the center of the disk the object starts to translate[14]. The temperature in the room is stabilized to $\mathrm{T}=20.0 \pm 0.5^{\circ} \mathrm{C}$.

The laser light diffracted by the asymmetrical printed object actually carries orbital angular momentum [13]. The conservation of the angular momentum leads to a reaction torque $\Gamma_{R}=N \alpha \hbar$, where $N$ is the number of photons incident on the rotating disk per second, and $\alpha$ accounts for the efficiency of the diffraction and the topological charge (i. e. the angular momentum carried per photon divided by $\hbar$ ) of the diffracted light. It depends on the relative size of the beam compared with the size of the printed picture, and on the degree of asymmetry of the printing. It is numerically evaluated from simulated diffracted light patterns[15] for each value of $p$ and for each printed picture size. For example, $\alpha=0.12$ for a $p=1$ paddle wheel, and a beam waist that corresponds to the paddle wheel size. It equals $\alpha=0.4$ and $\alpha=0.5$ for a $p=4$ and a $p=6$ paddle wheel respectively. $N$ 
depends on the optical power $P, N=P \lambda / h c, \lambda$ being the light wavelength and $c$ being the velocity of light. Note that this transfer is solely due to diffraction. It doesn't depend on the material used, on the environment, on the humidity, nor on the temperature of the liquid or of the disk.

Figure 3a shows the variation of the angular velocity $\omega$ of the disk versus light power for two different paddle wheels and two different disk diameters floating on the air/water interface. $\omega$ is extracted from the images using ImageJ software [16], from the temporal variation of the rotation angle. The reproducibility of the results is quite good as can be seen on the error bars obtained from several measurements. The laser power is measured at the end of the fiber. It doesn't vary throughout each experiment. As expected, the variation of $\omega$ versus light power is linear.

We have checked that $\omega$ doesn't depend on the surface tension (see figure 3b). Indeed, we have used several liquid solutions leading to different surface tensions. We have used either ethylene glycol $\left(\mathrm{C}_{2} \mathrm{H}_{6} \mathrm{O}_{2}\right)$ with percentage composition by mass $\mathrm{MC}=20$ and 50 leading to a surface tension $\gamma=55 \mathrm{mN} / \mathrm{m}$ and $\gamma=45 \mathrm{mN} / \mathrm{m}$ respectively[17], ammonium nitrate $\left(\mathrm{NH}_{4} \mathrm{NO}_{3}, \mathrm{MC}=65.5, \gamma=88\right.$ $\mathrm{mN} / \mathrm{m})[18]$, potassium carbonate $\left(\mathrm{K}_{2} \mathrm{CO}_{3}, \mathrm{MC}=24.5, \gamma=79 \mathrm{mN} / \mathrm{m}, \mathrm{MC}=33\right.$, $\gamma=82 \mathrm{mN} / \mathrm{m}$ ) $[17,18]$, sodium lauryl ether sulfate (SLES, MC=12, $\gamma=27$ $\mathrm{mN} / \mathrm{m})[19]$, or glycerol $\left(\mathrm{C}_{3} \mathrm{H}_{8} \mathrm{O}_{3}, \mathrm{MC}=25, \gamma=65 \mathrm{mN} / \mathrm{m}\right)[17]$. The surface tension has clearly no effect on the rotation of the disks. It has to be noted that the well-known surfactant sodium dodecyl sulfate (SDS) or the salt sodium chloride $(\mathrm{NaCl})$ at high concentrations strongly deteriorate the disks rapidly.

The accelerated regime lasts for a few milliseconds and is not detected, only the steady state rotation is observed. The rotation value is then constant within this regime. Relative fluctuations of the velocity are measured and are lower than 5\%. Let us evaluate the so-called Reynolds number for the highest rotation. $R_{e} \sim \rho R^{2} \omega / \eta$, where $\eta$ is the dynamical viscosity, and $\rho$ is the liquid density. 

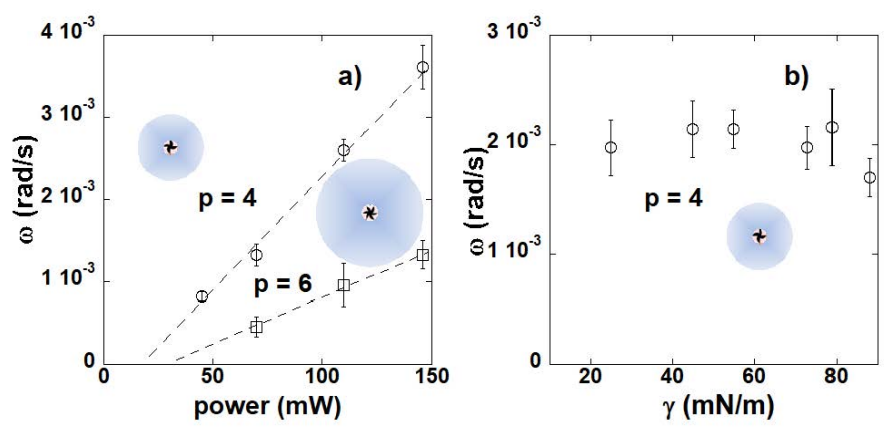

FIG. 3. Variation of the rotation velocity versus a) the light power for $p=4$ and $p=6$ printed pictures on different diameter size disks $(R=1.0 \mathrm{~mm}$ for $p=4$ and $R=1.5$ mm for $p=6$ ) (see drawing). b) The surface tension of the liquid for $p=4$ and $R=1.0$ $\mathrm{mm}$, for $P=100 \mathrm{~mW}$ (constant). The errors bars result from several measurements.

Taking $\eta \sim 10^{-3}$ Pa.s which is the value of water, $\rho=10^{3} \mathrm{~kg} / \mathrm{m}^{3}$, and $\omega=0.004$ $\mathrm{rad} / \mathrm{s}$, which is the highest value we found, leads to a value of $R_{e}<0.01$. This corresponds to a low Reynolds number. From the rotation of figure 3a, one can easily evaluate the drag coefficient inherent with this rotation. The expression of the drag torque $\Gamma_{D}$ for a cylindrical object of radius $R$ floating at the air/liquid interface and rotating at angular velocity $\omega$ with low Reynolds numbers flows, can be written [23]

$$
\Gamma_{D}=2 \pi k \eta R^{3} \omega
$$

where $k$ is a constant depending on the geometry of the object and the liquid motion[24]. The torque is perpendicular to the interface. Equating the expression of $\Gamma_{D}$ and $\Gamma_{R}$ which is the driving torque due to the light diffraction, leads to the following expression of the angular velocity

$$
\omega=\frac{P \alpha \lambda}{4 \pi^{2} c k \eta R^{3}}
$$

A linear adjustment of the variation of the rotation velocity versus the optical power of figure 3 a leads to slopes that equal $0.028 \mathrm{rad} . \mathrm{J}^{-1}$ and $0.0115 \mathrm{rad} . \mathrm{J}^{-1}$ for a $p=4, R=1.0 \mathrm{~mm}$ and $p=6, R=1.5 \mathrm{~mm}$ paddle wheels, respectively. Taking 
into account the differences for $\alpha$ and $R$ for the $p=4$ and $p=6$ paddle wheel, one can evaluate the drag coefficient $k \eta$ from equation (2). We measure the same drag coefficient $k \eta=1.25 \pm 0.2 \times 10^{-6}$ Pa.s for both cases. Compared with the usually assumed value of the drag coefficient[7, 23], $k \eta \simeq 5 \times 10^{-3}(k=16 / 3$ for an immersed disk, and $\eta \simeq 10^{-3}$ ), this value is abnormally small by more than three orders of magnitude. It has to be noted that we already had the same kind of unusual bulk viscosity with millimeter size objects[13]. We further investigate this phenomena by changing the viscosity of the solution, by adding glycerol. We have plotted the variation of the velocity versus the inverse of the bulk viscosity[20] (see figure $4 \mathrm{a})$.
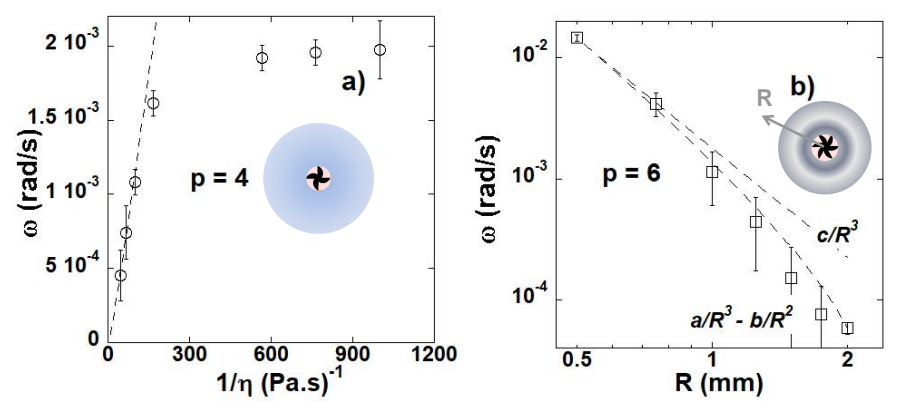

FIG. 4. Experimental variation of the rotation velocity versus a) the inverse of the usually assumed bulk viscosity for a $p=4$ and $R=1.0 \mathrm{~mm}$ paddle wheel. The disk radius is fixed. The dotted line corresponds to a linear fit. b) Disk radius $R$ for a $p=6$ paddle wheel in water. The different schemes of the disk illustrate the change of the disk radius. Note that both axis of the plot are on a logarithmic scale. The upper dotted line corresponds to an experimental fit of the data using Eq. 2 (c/ $R^{3}$ variation), whereas the lower dotted line corresponds to a fit according to Eq. 4 ( $a / R^{3}-b / R^{2}$ variation), $a, b$, and $c$ being constants. The experiments are performed at a power $P=100 \mathrm{~mW}$.

The variation of the angular velocity is linear for high viscosities and then saturates for lower viscosities. Although the linear variation is obtained on a very 
small viscosity range, let us focalize on the linear part. Its slope equals $10^{-5}$ Pa.rad, whereas the expected value from equation (2), for $P=100 \mathrm{~mW}, p=4$, $R=1 \mathrm{~mm}$, leads to $2 \times 10^{-9}$ Pa.rad. This confirms that the measured drag coefficient $k \eta$ corresponding to our set up doesn't match the usual value which is in the $10^{-3}$ Pa.s range. Besides the wetting properties are also correlated with the viscous dissipation [21]. This may also explain the saturation effect. Actually, adding some glycerol seems to induce an increase of the contact angle related to the roughness of the disk periphery. Then the drag coefficient increases, with a specific behavior related to fluid/solid interactions. It is worth noting that this cannot be explained by a change of the surface tension. Indeed, the addition of glycerol decreases the surface tension from $73 \mathrm{~N} / \mathrm{m}$ to $68.5 \mathrm{~N} / \mathrm{m}$ [22], which is too small to play any role. We also checked that there is no influence of the temperature of the liquid bath of the viscous dissipation.

The low value of the drag coefficient may have some consequences. Indeed, let us have a closer look at figure 4a. For the case of water (last point of the right), $\omega=2 \times 10^{-3} \mathrm{rad} / \mathrm{s}, R=1 \mathrm{~mm}$, and taking $k \eta=10^{-6}$ leads to a Reynolds number of $R_{e} \sim 2$. With a high glycerol concentration, $k \eta$ is multiplied by 10 , the velocity is divided by a factor of 4 . This leads to a Reynolds number $R_{e} \sim 0.05$. Although with $R_{e}<2$ the flow is still close to a laminar regime, could the plateau we observe on figure $4 \mathrm{a}$, be however due to a change of the flow regime, entering in a non-linear flow regime? This is actually not the case. In figure $3 \mathrm{a}$, when the optical power is varied, still taking the value of $k \eta=10^{-6}$, the Reynolds number decreases from $R_{e} \sim 3.5$ for $p=4, R=1 \mathrm{~mm}$ and $P=150 \mathrm{~mW}$ down to $R_{e} \sim 0.6$. We have even performed measurements with $R=0.5 \mathrm{~mm}$ rotating at $\omega$ varying from $10^{-2} \mathrm{rad} / \mathrm{s}\left(R_{e} \sim 2.5\right)$ to $2 \times 10^{-4}\left(R_{e} \sim 0.05\right)$ still showing a linear variation. If the flow would have entered a non-linear regime, the variation of the angular velocity vs the optical power wouldn't have been linear.

Could the low drag coefficient be due to the surface viscosity instead of the 
bulk viscosity[23]? A surface viscosity would lead to a drag torque varying as $R^{2}$, whereas a bulk viscosity would lead to a $R^{3}$ variation. We have thus varied the radius $R$ of the object for the same given $p=6$ printed paddle wheel and investigated the rotation velocity in water. The results are shown in figure $4 \mathrm{~b}$. For higher viscosities, the rotation was too slow and it was impossible to extract any drag coefficient. The Boussinesq number $B_{0}$ (dimensionless number representing the square root of the ratio of inertia force to gravitational force) is too low to have a frictional resistance of the surface film at the rim of the disk. Indeed we find $B_{0}=5 \cdot 10^{-4}$ at most. The experimental data better fits a $1 / R^{3}$ variation than a $1 / R^{2}$ one. The drag coefficient is not associated with a surface viscosity.

What could be the reason for this low viscosity value? A significant part of the light impinging on the object is absorbed raising up the temperature near the object. From the absorbed light power, we estimated this temperature to be at most around $60^{\circ} \mathrm{C}$ at the end of the measurement procedure. This has been estimated using a half-infinite space and a point heating on the air/water interface the by the laser. We overestimate the heating assuming that the total incoming power laser is absorbed. It is worth noting that we didn't see any condensation on the cover during experiments. The viscosity may decrease by a factor of two but not by three orders of magnitude.

On the other hand, the hydrophobic nature of the disk also contributes to the drag reduction [25]. Indeed, the disk is made of polyester (see figure 2). Its surface irregularities lead to the trapping of small air pockets between the object and the liquid layer (see figure 2 left part). We have seen that there are always some air micro-bubbles that remain at the interface between the disk and the liquid. The no-slip velocity condition between the solid and the liquid is thus no-longer fulfilled. At the interface, the liquid velocity is lower than the disk velocity. This no-slip condition may lead to a strong drag reduction. Indeed, the frictional drag between the solid and liquid interface may then be replaced by a much lower frictional drag 
between liquid and air[26, 27].

Besides, it is worth noting that the disk movement observed after $30 \mathrm{~s}$ for small disks may be due to the heating of these air micro-bubbles, leading to the translational motion already mentioned. One can calculate the thermal resistance of the polyester disk. Its thermal conductivity is about $0.1 \mathrm{~W} \cdot \mathrm{m}^{-1} \cdot \mathrm{K}^{-1}$. For a thickness of $187 \mu \mathrm{m}$, the thermal resistance is about $104 \mathrm{~K} . \mathrm{W}^{-1}$ for a typical size of $500 \mu \mathrm{m}$ of the paddle wheel. For a laser power of $100 \mathrm{~mW}$, the temperature rise between the disk and the air/liquid interface during 3 min exposure time should be at most $2 \mathrm{~K}$. For shorter times, the heating has then nearly no influence otherwise we wouldn't have been able to evidence a linear variation of the rotation velocity versus laser power (see figure 4a). Besides, when the laser is switched on, the temperature of the disk is the same as the temperature of the water and then rises with time. We observe a steady state rotation, with a constant velocity, showing that this heating has indeed little influence. Moreover, concerning the drag coefficient, the edges of the disk are not so regular and seem jagged at the micron scale, due to the punching process. Of course we could have used a highly hydrophilic disk $[7,23]$ to perform this kind of experiment, as it is usually done in micro-rheological studies. However, the wetting properties promote the bulk viscosity contribution and thus prevent us from having access to the very rotation regime we observe here.

Other effects that are usually hidden may then play a significant contribution. In particular, the adjustment performed on figure $4 \mathrm{~b}$ leads to a good fitting curve for the lowest values of $R$, whereas there is a discrepancy for higher values of $R$. Clearly, the fit is out of the experimental error bars for the last three points. Although there is a rather high relative uncertainty on the experimental data, the adjustment using a regression analysis fits much better with a $1 / R^{3}$ and a $1 / R^{2}$ varying terms then for a $1 / R^{3}$ alone, as it can be clearly seen on figure $4 \mathrm{~b}$. Even though the fit is not perfect we may estimate a reliability factor. It equals 0.97 
for a $1 / R^{3}$ fit and increases to 0.999 adding a $1 / R^{2}$ term. The rotation velocity $\omega$ scales as $\omega=2 \times 10^{-12} / R^{3}-9 \times 10^{-10} / R^{2}$. The units of the pre-factor of the first term is $\operatorname{rad} . \mathrm{m}^{3} / \mathrm{s}$, where it is $\mathrm{rad} . \mathrm{m}^{2} / \mathrm{s}$ for the second. From Eq. 2, the first term leads to a value of the drag coefficient $k \eta=1.45 \pm 0.2 \times 10^{-6} \mathrm{~Pa}$.s, in very good agreement with the value deduced from the linear dependence of the angular velocity versus the optical power (see figure 3a). Nevertheless, one has to explain the origin of the extra term.

Actually, in the physical problem of a floating object on a liquid interface, like the one considered here, there is a three phase contact zone (circumference of the disk at the water/air/solid contact line) corresponding to a one dimensional curve. Then the so-called line tension may have an influence. The line tension concept was proposed by Gibbs more than a hundred years ago and it is associated with the excess of free energy of a solid-liquid-vapor system per unit length of contact line or with the excess of force acting along the three-phase line to shorten or lengthen it[28]. The line tension depends on the object wettability as well as on the shape of the air/liquid interface near the contact line. However, it is not correlated to the surface tension [29]. Besides, this quantity is usually too small to have practical significance[28], or to play any role. Indeed, the true value of the line tension and its sign remains disputable. De Gennes et al. even wrote about the "mythos of line tension" [30], and the literature reporting theoretical and experimental investigations is very broad. Yet, since the drag coefficient $k \eta$ is very low here, one may have experimentally access to a measurement of this quantity. Besides, since the typical diameter size is of the order of the capillary length $\lambda_{c}\left(\lambda_{c} \sim 2 \mathrm{~mm}\right)$, the line tension must play a role in our experiment.

Let us then introduce the line tension force $\tau$ on the rotating disk. It is tangent to it and opposed to the rotation. The expression of the torque due to this line tension then writes $\Gamma_{T}=2 \pi \tau R$. It is orientated perpendicular to the interface. It thus can play a role in the dynamics of the disk rotation. As recently suggested 
for the study of contact angle hysteresis [31], the energy needed to deform the line contact for a rotation of an angle $d \theta$ of the notched disk writes $d E=\Gamma d \theta$, where $\Gamma$ is the torque needed. Then the optical energy transferred to the disk movement can be decomposed into the rotational kinetic energy and the work of the line tension force. The steady state equation of the rotation of the disk, including the line tension torque now reads

$$
\Gamma_{R}=\frac{P \alpha \lambda}{2 \pi c}=\Gamma_{D}+\Gamma_{T}=2 \pi k \eta R^{3} \omega+2 \pi \tau R
$$

The effect of the line tension could be compared with a dry friction for solid surfaces, where one defines a static and a kinetic friction coefficient. This leads to a variation of the angular velocity that writes

$$
\omega=\frac{P \alpha \lambda}{4 \pi^{2} c k \eta R^{3}}-\frac{\tau}{k \eta R^{2}}
$$

and finally leads to a value of $\tau=1.3 \pm 0.3 \times 10^{-15} \mathrm{~N}$, i.e. $\tau \sim 10^{-15} \mathrm{~N}$, which is rather low [28].

Nevertheless, let us have now a closer look at figure 3a. Obviously, both curves don't cross the origin. There is a small shift of the curves or threshold, corresponding to $P=35 \mathrm{~mW}$ and $P=25 \mathrm{~mW}$, for a $p=6$ and a $p=4$ paddle wheel, respectively. When we tried to measure the rotation velocity at those powers, we could estimate that the rotation is less than $2^{\circ}$ in 6 minutes. We therefore assume that it is equal to zero. Setting $\omega=0$ in equation (4) leads to $\tau=1.3 \pm 0.1 \times 10^{-15}$ $\mathrm{N}$ and $\tau=0.84 \pm 0.06 \times 10^{-15} \mathrm{~N}$, respectively. These values are in good agreement with the value of $\tau \sim 10^{-15} \mathrm{~N}$ deduced from figure $4 \mathrm{~b}$. This threshold indeed corresponds to the torque generating light power that is needed to counter balance the line tension $\tau$.

Below this value, the disk does not rotate, and, since the line tension is defined as a force, Eq. 3 is no more valid. This is the same situation observed in the case of dry friction for which there is no movement of the solid below a threshold force. A similar description has been recently proposed to explain the sliding of liquid 
drops on a solid surface [31]. Beyond this value, the rotation rate increases linearly with the power, minus the constant term due to the line tension. It validates the model we used, taking into account the line tension, and equation (4) as well. This leads to a line tension coefficient in the femto Newton range in the case considered here. Not that the value we measured is necessarily positive since it is opposed to the rotation, otherwise the disk would have started to rotate on its own, which has physically no meaning.

As a conclusion, we have transferred angular momentum from a plane wave to a disk, because of the diffraction from an asymmetrical picture printed on the disk. This enables the evaluation of a drag coefficient of the liquid. Since the object is hydrophobic, and because there are many micro-air bubbles and the air/liquid interface, this viscosity is unexpectedly low, in the $10^{-6}$ Pa.s range for pure water. We are then able to evidence the role of the line tension and the existence of a threshold value corresponding to the counterbalance power needed to compensate for this line tension. Although our study does not enable us to give a precise value of the line tension, it shows that its contribution related to the heterogeneities in the vicinity of the contact line is not negligible. The order of magnitude of this line tension is in the $10^{-15}$ range. Of course these findings deserve confirmation at smaller scales, close to the micrometer range and further theoretical investigations and calculations to explain these findings. Nevertheless, the technics we developed here clearly opens new avenues towards line tension measurements, and precise micro-rheological measurements in general[32].

Acknowledgments. The authors would like to thank Julien SINFELT for early interest and Stéphane BOUTROS and Michel BELLIOUS for technological support and valuable help. 


\section{CONFLICT OF INTEREST}

There are no conflicts to declare.

* Corresponding author; olivier.emile@univ-rennes1.fr

[1] D. G. Grier, Nature, 2003, 424, 810.

[2] K. Kim, J. Guo, Z. X. Liang, F. Q. Zhu, and D. L. Fan, Nanoscale, 2016, 8, 10471.

[3] J. W. Judy, Smart Mater. Struct., 2001, 10, 1115.

[4] J. Leach, H. Mushfique, R. di Leonardo, M. J. Padgett, and J. Cooper, Lab Chip, $2006,6,735$.

[5] J. Lipfert, M. M. van Oene, M. Lee, F. Pedaci, and N. H. Dekker, Chem. Rev., $2015, \mathbf{1 1 5}, 1449$.

[6] M. Safdar, J. Simmichen, and J. Jänis, Environ. Sci. Nano, 2017, 4, 1602.

[7] A. I. Bishop, T. A. Nieminen, N. R. Heckenberg, and H. Rubinsztein-Dunlop, Phys. Rev. Lett., 2004, 92, 198104.

[8] V. L. Calero-DdelC, D. I. Santiago-Quinonez, and C. Rinaldi, Soft Matter, 2011, 7, 4497.

[9] P. Galajda and P. Ormos, Appl. Phys. Lett., 2002, 80, 4653.

[10] M. E. J. Friese, T. A. Nieminen, N. R. Heckenberg, and H. Rubinsztein-Dunlop, Nature, 1998, 394, 348.

[11] H. He, M. E. J. Friese, N. R. Heckenberg, and H. Rubinsztein-Dunlop, Phys. Rev. Lett., 1995, 75, 826.

[12] A. M. Yao and M. J. Padgett, Adv. Opt. Photon., 2011, 3, 161.

[13] O. Emile and J. Emile, Opt. Lett., 2016, 41, 211.

[14] M. Škarabot, N. Osterman, and I. Muševič, Soft Matter, 2017, 13, 2448.

[15] O. Emile, M. le Meur, and J. Emile, Phys. Rev. A, 2014, 89, 013846.

[16] C. A. Schneider, W. S. Rasband, and K. W. Eliceiri, Nat. Methods, 2012, 9, 671. 
[17] Å. Melinder, PhD Thesis, KTH Royal Institute of technology Stockholm, 2007.

[18] C. S. Dutcher, A. S. Wexlerand, and S. L. Clegg, J. Phys. Chem. A, 2010, 114, 12216.

[19] F. Comelles, I. Ribosa, J. J. González, and M. T. Garcia, J. Colloid Interface Sci., $2014, \mathbf{4 2 5}, 44$.

[20] J. B. Segur and H. E. Oberstar, Ind. Eng. Chem., 1951, 43, 2117.

[21] N. Selway, V. Chan and J. R. Stokes, Soft Matter, 2017, 13, 1702.

[22] K. Takamura, H. Fischer, and N. R. Morrow, J. Pet. Sci. Eng., 2012, 98-99, 50.

[23] Z. A. Zell, A. Nowbahar, V. Mansard, L. G. Leal, S. S. Deshmukh, J. M. Mecca, C. J. Tucker, and T. M. Squires, Proc. Natl. Acad. Sci., 2014, 111, 3677.

[24] F. C. Goodrich, and A. K. Chatterjee, J. Colloid Interface Sci., 1970, 34, 36.

[25] N. Murazawa, S. Juodkazis, V. Jarutis, Y. Tanamura, and H. Misawa, EPL, 2006, $\mathbf{7 3}, 800$.

[26] J. P. Rothstein, Annu. Rev. Fluid Mech., 2010 42, 89.

[27] Y. L. Zhang, H. Xia, E. Kim, and H. B. Sun, Soft Matter, 2012, 8, 11217.

[28] B. V. Toshev, D. Platikanov, and A. Scheludko, Langmuir, 1988, 4, 489.

[29] M. Kanduč, J. Chem. Phys., 2017, 147, 174701.

[30] P. G. De Gennes, F. Brochard-Wyart, and D. Quéré, Capillarity and wetting phenomena, Springer, Berlin, 2003.

[31] L. Makkonen, J. Chem. Phys., 2017, 157, 064703.

[32] P. Cicuta and A. M. Donald, Soft Matter, 2007, 3, 1449. 